\title{
Influence of Cognitive Factors on Interpreters' Performance
}

\author{
Mianmian Cai \\ Xiamen University Tan Kah Kee College \\ Zhangzhou, China
}

\begin{abstract}
This thesis mainly presents the application of interdisciplinary research to dynamic study of interpretation, i.e. the study of the process of interpretation. The author discussed Relevance Theory, which is known as theoretical foundation of cognitive pragmatics. After explaining the procedure of comprehension, the author provides some examples to show the importance of finding a shared cognitive environment.
\end{abstract}

Keywords-relevance theory; interpreting; cognitive pragmatics

\section{INTRODUCTION}

Since languages are often obstacles for interlingual and intercultural communication, translation becomes a gobetween to bridge the language gap. Translation is to express the meaning of one language by another language. It has two forms, oral interpretation and written translation [1]. In spite of the fact that both translation and interpretation belong to the category of translation, interpretation has some distinct features when compared with translation.

First of all, what the interpreter first comes across in interpretation is the phonetic symbols which stimulate his sense of hearing. However, it is word symbol that stimulates the translator's vision in translation. So we say, the ways the interpreter and translator receive the information at the initial stage are different. Because the words or sentences of the speech in interpretation are evanescence, if the interpreter stops at certain words, it may lead to the loss of information [2]. Primarily, the interpreter is expected to excel at listening comprehension ability. Not all speakers speak Standard English. According to an informal survey, half of the speakers' mother tongue is not English. Therefore, interpreters should equip themselves with the ability to understand the language with or without accents.

Secondly, interpretation is an extemporaneous activity. When confronted with difficulties, the translator can consult dictionaries or refer to some information books such as references and other available materials or even turn to the writer for suggestions, if possible, but the interpreter has no chance to do that, because the interpreter must process the speaker's information and deliver the interpretation in the target language fluently within a very short time. In addition, the speakers who the interpreter serves are probably from different ranks of society, different walks of life and especially different cultural backgrounds. Therefore their speeches have no bound, covering a rather wide range of subjects. Along with this variety and infinity of speech in the course of interpretation, the interpreter has to constantly go through very arduous and complicated thinking processes, which requires the essential participation of cognitive knowledge so as to interpret the input information in a proper way by relating to relevant knowledge. To carry out the interpretation study, the study from the cognitive perspective cannot be neglected. The combination of cognitive science and pragmatics, i.e. cognitive pragmatics, has provided a new cognitive perspective for the study of interpretation. As a matter of fact, cognitive pragmatics has been drawing more and more attention of the academia since the 1980s. This new approach to the study of natural language use, with its focus on speaker meaning and utterance interpretation, has shed some light on interpreter's comprehension and reproduction process.

\section{AN OVERVIEW OF INTERPRETATION STUDIES IN CHINA}

China has a late start in interpretation studies. Chinese researchers and scholars did not begin to explore this recondite art until the early 1980s. Compared with its status in the west, the study of interpretation is a new discipline in China. It did not break away from its stagnant period until the 1980s when the first articles on interpretation were published, among which most were based on interpreters' professional experience, introspection and so on. With the rapid growth of domestic economy and increase of external exchanges, interpretation studies have drawn people's attention.

Interpretation embodies both static meaning and dynamic meaning. Correspondingly, the studies of interpretation fall into two categories: static study and dynamic study. Static study generally focuses on the quality assessment, criterion, etc. of the product of interpretation, which have laid a solid foundation for interpretation criticism. However, when it comes to the key to successful interpretation, static study fails to deliver the answer, because it neglects the fact that during interpretation, a high level of linguistic competence is not the only factor that counts. Aimed at conveying information between two or more parties, interpretation is an interactive behavior and therefore is a kind of dynamic communicative act; it involves social context. According to 
Jean - Claude Abric, there are three main elements that influence communication: psychological factor, cognitive factor and social factor [3]. Adaptive theory also claims that as a social act, the use of language is closely related to psychological factor, cognitive factor and social factor [4]. An attempt will be made to discuss the cognitive factor in this paper. Among all the branches of cognitive pragmatics, Relevance Theory (hereinafter referred to as "RT"), known as the theoretical foundation of cognitive pragmatics, has exerted a great influence on the western academic community. This theoretical framework will now be discussed so as to give the readers a brief introduction of the general idea of this theory.

\section{RELEVANCE THEORY: A NEW APPROACH TO INTERPRETATION}

RT is put forward by English scholar Dan Sperber and French scholar Deirdre Wilson in their book Relevance: Communication and Cognition first published in 1896 and then updated in 1995. Since the study on interpretation theory in the perspective of cognitive pragmatics will be carried out in the light of RT, the author believes that a general introduction of some of its central concepts, such as relevance, ostensive stimulus, the Cognitive Principle and the Communicative Principle, will make RT more comprehensible to readers.

\section{A. The Definition of Relevanc and the Comprehension Procedure}

There are two communication models: code model and inferential model. Through the classical code model, a communicator encodes her intended message into a signal, which is then decoded by the audience using an identical copy of the code. Sperber and Wilson hold that the code model is subordinate to the inferential model, which is fundamental in the course of communication. According to the inferential model, a communicator provides evidence of his intention to convey a certain meaning, which is inferred by the audience on the basis of the evidence provided. An utterance is, of course, a linguistically coded piece of evidence, so that verbal comprehension involves an element of decoding.

How does the audience infer the communicator's meaning from the evidence provided? RT regards communication as an ostensive-inferential process. Standing in communicator's position, communication is an ostensive process in that the communicator manifests his intention to the audience in an ostensive way, i.e. using an ostensive stimulus. When people communicate, to speak to someone is an ostensive behavior which provides evidence of one's thoughts. An ostensive stimulus is designed to attract the audience's attention and focus it on the communicator's meaning. RT claims that the use of an ostensive stimulus may create precise and predictable expectations of relevance not raised by other stimuli. By producing an ostensive stimulus, the communicator therefore encourages the audience to presume that it is relevant enough to be worth processing.
Then the audience draws an inference from an appropriate set of contextual assumptions. The audience supplies contextual assumptions on the basis of three information of cognitive environment: logical information, encyclopedic information and lexical information. In order to make certain the implicit message of the communicator and comprehend the communicator's intention, the audience should make some efforts to seek relevance between the two kinds of information, the new information formed by stimulus and the old assumptions made before. Intuitively, relevance is a potential property not only of utterances and other observable phenomena, but of thoughts, memories and conclusions of inferences. In relevance-theoretic terms, an input is relevant to an individual when its processing in a context of available assumptions yields a positive cognitive effect. The higher the relevance of the assumption to the hearer at that time, the less processing effort expended, the better cognitive effects achieved. The process of inference is the process of finding optimal relevance.

Sperber and Wilson summarized the Relevance-theoretic comprehension procedure as follows:

- Follow a path of least effort in computing cognitive effects: Test interpretive hypotheses (disambiguations, reference resolutions, implicatures, etc.) in order of accessibility.

- Stop when your expectations of relevance are satisfied.

In terms of RT, the audience has to make some efforts to find out the meanings of the communicator's utterance. The audience uses a general principle to evaluate meanings. This criterion is powerful enough to exclude all but at least one single meaning would satisfy it. This is the basis for RT's two fundamental principles: Cognitive Principle of Relevance and Communicative Principle of Relevance. Cognitive Principle is a reasonable explanation of human cognition which is underlying mechanisms rooted in human psychology. Communicative Principle is an explanation of communicative law which makes clear how humans communicate with one another. Every aspect of cognition and communication is governed by the search for relevance. These two principles will be discussed in the following.

\section{B. The Cognitive Principle of Relevance}

Sperber and Wilson define Cognitive Principle of Relevance as "Human cognition tends to be geared to the maximization of relevance" [5]. It is also called the First Principle of Relevance, presenting the basic assumption about human cognition.

RT claims that humans do have an automatic tendency to maximize relevance because, owing to the constant selection pressure towards increasing efficiency, the human cognitive system has evolved in such a way that our perceptual mechanisms tend automatically to select potentially relevant stimuli, our memory retrieval mechanisms tend automatically to activate potentially relevant assumptions, and our inferential mechanisms tend spontaneously to process them in the most productive way. Thus, while we are 
all likely to notice the sound of glass breaking in our vicinity, we are likely to attend to it more, and process it more deeply, when our memory and inference mechanisms identify it as the sound of our glass breaking, and compute the consequences that are likely to be most worthwhile for us[6].

It is against this cognitive background that inferential communication takes place.

\section{The Communicative Principle of Relevance}

By producing an ostensive stimulus, the communicator therefore encourages her audience to presume that it is relevant enough to be worth processing. This is the basis for the Second, or Communicative, Principle of Relevance, which applies specifically to ostensive-inferential communication:

Sperber and Wilson define Communicative Principle of Relevance as "Every ostensive stimulus conveys a presumption of its own optimal relevance." [5].

From the definition, it can be concluded that Communicative Principle is concerned with the optimal relevance for which people seek in communication. According to RT's definition of optimal relevance, an ostensive stimulus is optimally relevant to an audience:

- It is relevant enough to be worth the audience's processing effort;

- It is the most relevant one compatible with communicator's abilities and preferences.

Communicative Principle is also named Principle of Relevance or the Second Principle of Relevance. This inferential theory of communication is the most basic claim of RT.

\section{Enlightenment of Relevance Theory on Interpreters' Performance}

With a preliminary knowledge of RT and cognitive environment, we may draw some useful lessons for interpretation study in light of RT.

When the primary speakers want to communicate with each other, both of them search for relevance based on their own cognitive environments. However, in the process of interpretation, the search for relevance is realized by the interpreter due to the gap of languages and cultures. Since the interpreter is in a secondary communicative environment, his cognitive environments are independent from those of the primary communicators. When comprehending and reproducing the speakers' informative intention and communicative intention, the interpreter inevitably employs his own contextual assumptions. Undoubtedly, this leads to a discount of relevance. When receiving information from the original speaker, the interpreter should seek for the context as close as that of the original speech and relate the new information to his cognitive environments to infer the speaker's communicative intention; when sending information to the target hearer, the interpreter has to take into consideration the target hearer's cognitive environments and offer them the reproduction with optimal relevance.
Successful interpretation depends to a large extent on the transfer of optimal relevance from the source text to the target text. Since the interpreter stands at the center of the communication, his choice of cognitive environment is of great significance. If the contextual assumptions the interpreter employs can be as close as possible to those of the primary communicators, then the discount of relevance can be remarkably offset.

Consequently, the precondition of the interpreter's successful search for optimal relevance lies in that the interpreter should be equipped with adequate contextual knowledge which is ready to be retrieved at any time. Just as the author has mentioned above, the cognitive environment consists of not only logical information and lexical information, but also encyclopedic information, which , standing in the interpreter's position, includes the background of both the speaker and the hearer, knowledge of relevant subject, terminology, etc. Since the cognitive environment which constitutes assumptions differ from person to person, people with different cognitive environments may get different meaning of the same thing. However, the Principle of Relevance can greatly reduce misunderstanding. Relevance guides interpretation. Therefore, when the interpreter makes an inference of the speaker's intention, he should manage to expand his cognitive environment so as to make it as close as that of the speaker. This preexistent knowledge of the interpreter builds language prediction module. If the interpreter constantly activates this knowledge, which will later constitute potentially relevant assumptions, then when it comes to actual interpretation work, the interpreter will be able to pick up the relevant knowledge from his brain in a quick and accurate way, cut down assumptions of low relevance and ultimately reach the optimal relevance. In addition, the quicker the interpreter finds the optimal relevance, the less effort expended. In this way, the interpreter can save his processing efforts for language production.

On the contrary, if the interpreter lacks the necessary relevant encyclopedic knowledge, such as unfamiliar subject matters and words, cultural differences, it will be rather difficult for him to draw assumptions, which may lead to disconnection with relevance. To further prove this point, the author brings machine translation into comparison. The popularization of translation software and websites has to some extent facilitated working efficiency of translators and interpreters, due to their rich vocabulary and updated words and expressions. However, such situations happen to the users of machine translation every now and then that the translation by machine is awkward and illogical. The reason mainly lies in that the machine translates on the basis of syntactic analysis. Its scarcity of encyclopedic knowledge makes it incapable of drawing contextual assumptions, not to speak of cutting down assumptions of low relevance and ultimately reaching the optimal relevance. Here is an example: Gas pump prices rose last time oil stocks fell. Every word in this sentence can be used as a noun and a verb. It is hard to imagine how many sets of combination the machine has to make in order to arrive at the correct translation, if it may. Just as Professor Lin Yuru claims, 
"Comprehension occurs only when information ties in with related knowledge. If this knowledge is absent, the new information cannot be understood or assimilated, for the learner has nothing to relate the new knowledge to."[7]. For example, during a medical conference, the interpreter encounters the abbreviation "EMS". If he does not possess the required medical knowledge, he may be inclined to comprehend it as Express Mail Service, which he will shortly realize not related to the topic and end up in confusion. Instead, if the interpreter acquaints himself with the subject, he may understand the abbreviation properly as Electronic Medical System.

As for reproduction, the interpreter should faithfully convey the speaker's intention to the audience. According to the conditions for optimal relevance, the optimal interpretation is produced on the basis of the greatest contextual effect and the least processing effort. Thus, the interpreter should also infer the cognitive environments and anticipation of the audience. RT holds that relevance is conceived as relative or subjective, as it depends upon the state of knowledge of the audience when they encounter an utterance. So, due to difference in people's physical environment, experience, cognitive abilities, etc. the apparent information in the original context may turn out to be indigestible knowledge in the target context. Here are some examples.

1、我们一定会站在历史和时代的高度, 努力推进中国高等教育的 国际化和特色化。

“历史和时代的高度” is a typical unspecific, abstract Chinese concept. The word “高度” does not have practical meaning, which makes it difficult to connect with the English speakers' cognitive environment and therefore needs to be made concrete.

Suggested interpretation: "Our nation will make serious plans for internationalized and characteristic higher education from a historic perspective."

2、各级预算都要量入为出, 一是吃饭, 二是建设。

If “吃饭” is interpreted literally as "having meals", then the audience may be confused about Chinese people's obsession with food. Evidently the word's implications go beyond having meals. If we take the context into consideration which lays emphasis on discussing budget, then we will see the implications of the phrase cover a wide range. Therefore, the phrase may be interpreted as "subsistence".

Suggested interpretation: "In preparing their budget, governments at all levels should make sure that they can make both ends meet and that budged expenditures cover both subsistence and development costs." 来。

3、他这一阵心头如同十五个吊桶打水, 七上八下, 老是宁静不下

The Chinese culture is known for its implicit style. The feeling of the character in this sentence is expressed indirectly as "fetch water with fifteen buckets, seven buckets up and eight buckets down". If the sentence is interpreted literally, it will undoubtedly be very difficult for the audience to picture the image, not to mention understanding the fourcharacter Chinese idiom which refers to an unsettled state of mind. Therefore, to help the audience find the optimal relevance, it is better to interpret this sentence in a direct way according to its real meaning so that the speakers' informative intention and communicative intention can be reproduced.

Suggested interpretation: "His mind was in turmoil these days and he was quite unable to think straight."

4. Clearly you in China have understood, as your saying goes, that we all "share the same breath". Human misery knows no frontiers, and nor should human solidarity.

Since one of the distinctive features of the Chinese language is symmetry in form which also embodies the aesthetical function, it accords with the Chinese way of expression to add “共命运” to “同呼吸”, without which the interpretation may sound a little strange to the audience.

Besides, to use two phrases with the same structure makes it more powerful.'

Suggested interpretation: “正如你们所说的“同呼吸共命 运, , 中国人民显然深知其义。人类的苦难没有国界, 我们也应同样 不分国界地区地团结在一起”。

\section{5、加强基层医疗卫生服务体系。}

When it comes to the interpretation of “基层” , it is often the case that some interpreters immediately come up with the word "grassroots". However, in English, "grassroots" refers to the common people as opposed to the people with power the authorities. So, in accordance with the political system of China, it will be much better to interpret “基层” into "community" or "primary level" so that the speaker's informative intention will not be misunderstood.

Suggested interpretation: "improve community-level healthcare services"

\section{6、宣传党的主张}

For decades, “宣传” has been interpreted as "propaganda" or "publicity". The intention of the former is to influence people's opinions and the latter to attract people's interest or attention, both with negative implications. This interpretation will make people question speaker's sincerity and distort the speaker's informative intention and communicative intention. It is necessary to avoid misunderstanding.

Suggested interpretation: "communicating the Party's propositions"

7、中华民族伟大复兴, 绝对不是轻轻松松、敲锣打鼓就能实现 的。

The phrase “敲锣打鼓” can be literally interpreted as drum beating and gong clanging. However, it may be difficult for the audience to thoroughly understand the implications. Actually, the phrase conveys the meaning that achieving China's rejuvenation is not an easy job and cannot be accomplished simply by exclaiming slogans. The sentence shows Chinese people's determination that all thinking and behavior in the vein of pleasure seeking, 
inaction and sloth, and problem avoidance are unacceptable. Consequently, it is vital to accurately deliver the information that achieving China's rejuvenation is a difficult and challenging journey and Chinese people are prepared to work hard toward this goal. In this case, "walk in the park" is an excellent equivalent. The target audience can immediately relate to the speaker's informative intention and communicative intention.

Suggested interpretation: Achieving national rejuvenation will be no walk in the park; it will take more than drum beating and gong clanging to get there.

In a word, playing the dual interactive role of message receiver and message sender, the interpreter is responsible for finding a shared cognitive environment between the two parties. The interpreter should carefully select proper words and expressions so that the audience' cognitive environment can be as close as that of the speaker.

\section{CONCLUSION}

This paper is a tentative study of interpretation under the guidance of relevance theory. As stated in RT, human cognition is relevance-oriented and human communication is an ostensive-inferential process governed by the search for optimal relevance.

It indicates that in the process of comprehension, the interpreter should search for the context that is closely relevant to the information conveyed in the utterance; and in the process of reproduction, he should also consider the target hearer's cognitive environment so as to achieve optimal relevance. This relevance-theoretic account of cognition and communication has practical implications for the study of interpretation theory.

As international communication takes place more frequently, interpreting ascends to a more significant status, so do interpretation studies. The significance of theory's guidance to practice can never be neglected. The nature of interpretation determines interdisciplinary research on it is the unavoidable trend. Interpretation is a complicated cognitive activity for the purpose of cross-cultural communication involving transfer of oral information from one language to another. Studies on interpretation inevitably cross various related fields such as cognitive psychology, sociolinguistics, psycholinguistics, linguistics, and so on.

In the communication process, interpreter works as "a mediator standing at the centre of the dynamic process of communication between the SL speaker and TL audience"[8] Interpreting is a very complicated and particular dynamic commutative act. All factors are always in dynamic state. The cognitive factor, in particular, constantly varies, exerting a direct or indirect influence on the result of interpreting. Knowledge of the cognitive factor is essential to interpreters' improvement.

Furthermore, to find the optimal relevance from the source context, the interpreter needs to be familiar with the thinking way of the people of the source culture and manage to offset the difference of thinking ways between two cultures. After all, interpretation is the conformity of language cognition and culture cognition.

The author sincerely hopes that a preliminary discussion of this dynamic communicative act and the major influencing factors will help interpreters to strategically select interpretation based on these factors, so as to convey the genuine intention of the source text.

\section{REFERENCES}

[1] Mei Deming, An Advanced Course of Interpretation Shanghai: Shanghai Foreign Language Education Press, 2000, p.3.

[2] Liu Heping, Theory and Teaching of Interpretation. Beijing: China Translation Corporation, 2005, p. 12.

[3] Jean-Claude Abric, Psychology of communication. Methods and Theory. Paris: Masson \& Armand Collin, 1996.

[4] Zeng Wenxiong, "Linguistic application during interpretation" in Journal of Changchun Normal University, vol. 22, p106, 2003.

[5] Sperber \& Wilson. Relevance: Communication and Cognition, 2nd ed., Beijing: Foreign Language Teaching and Research Press, 2001, p. 260.

[6] Sperber \& Wilson. Relevance: Communication and Cognition, Oxford: Blackwell, 1995.

[7] Lin Yuru, Qu Guowen and Zhuang Hongshan, New Course in Spoken English. Xiamen: Xiamn University Press, 1987.

[8] Hatim, B \& Mason, I. Discourse and the Translator. Shanghai: Shanghai Foreign Language Education Press, 2001. 